Apidologie, 1982, 13 (2), 127-141.

\title{
PRÄPARATVERTEILUNG BEI SYSTEMISCHER THERAPIE VON EKTOPARASITOSEN BEI APIS MELLIFERA L.
}

\author{
Robin F. A. MORITZ \\ Institut für Bienenkunde (Polytechnische Gesellschaft) \\ der J.W. Goethe Universität Frankfurt/Main \\ Fachbereich Biologie, Im Rothkopf 5 \\ 6370 Oberursel/Ts., F.R.G.
}

\section{ZUSAMMENFASSUNG}

Bei der systemischen Therapie von Ektoparasitosen bei Bienen ist der Effekt eines Präparates von der Verteilung im Bienenvolk abhängig. Mit einer Phosphor ${ }^{32}-$ und einer Methylenblaumarkierung wurde die Präparatverteilung bei verschiedenen Trägermedien und verschiedenen Volumina in Sommer- und Wintervölkern gezeigt. Eine systemische Therapie mit mehrmaliger Applikation kleiner Volumina in Wintervölkern erweist sich am effektivsten.

\section{EINLEITUNG}

Bei einer systemischen Chemotherapie von Ektoparasitosen wird ein Wirkstoff vom Wirtsorganismus resorbiert und über die Körperflüssigkeit des Wirts auf den saugenden Parasiten übertragen. Das Präparat sollte eine möglichst geringe wirtspathogene Wirkung zeigen, aber einen deutlich parasitiziden Effekt aufweisen. Aus dem Pflanzenschutz sind eine Reihe solcher systemischer Therapeutika bekannt, die über die Leitgefässe in der gesamten Pflanze verteilt werden. Saugende Schädlinge werden somit spezifisch, weitgehend ohne Schädigung der bestäubenden Insekten durch Kontaktwirkung, erreicht. Bei der Honigbiene (Apis mellifera L.) liegt im Falle einer systemischen Therapie ein ähnlicher Wirkmechanismus vor. Das entsprechende 
Präparat wird mit dem Futter von der Biene aufgenommen und resorbiert. Über die Haemolymphe wird das Präparat vom Parasiten aufgenommen. Im Falle der Honigbiene gewinnt eine systemische Wirkung besondere Bedeutung, da innerhalb des Bienenvolks über die Trophallaxe eine kleine Präparatmenge schnell im gesamten Bienenvolk verteilt wird. NiXON und RiBBands (1952) konnten in ihrer klassischen Arbeit zur Verteilung eingetragenen Futters im Bienenvolk mit einem Phosphor ${ }^{32}$ Tracer zeigen, wie schnell markiertes Zuckerwasser von den Sammelbienen an die Stockbienen verteilt wird. GösswaLD und KLOFT (1958) konnten in Laborversuchen nachweisen, dass das Futter einer Arbeiterin an bis zu 50 Nestgenossinnen weitergegeben werden kann. Dies verdeutlicht die grosse Bedeutung, die systemische Präparate in der Therapie von Bienenkrankheiten spielen können.

Erste Ergebnisse einer systemischen Therapie von Ektoparasitosen der Honigbiene liegen seit den Arbeiten von KüNzLER (1980) und RUTTNER et al. (1980) mit dem Wirkstoff Chlordimeformhydrochlorid (K-79) vor. Dieses Präparat wird zur Therapie der Varroatose, einer Milbeninfektion der Honigbiene, als systemisches Akarizid eingesetzt. Das Mittel zeigt eine hohe Milbentoxizität bei geringer Bienengefährlichkeit (RADEMACHER, 1980; MoRTT, 1980). Es galt eine Applikationsweise zu finden, die die Prämissen einer jeden Chemotherapie zu erfüllen hat. Bei maximaler Akarizidität und tolerierbarer Bienenmortalität sollte insbesondere im Falle einer Therapie mit humantoxischen Präparaten der Rückstand im Honig minimiert werden. Neben den pharmakologischen Eigenschaften des Präparates wird dabei die Verteilung im Bienenvolk einen entscheidenden Einfluss auf den Therapieerfolg haben. Für die Mitteldistribution erscheinen folgende vier Parameter von besonderer Bedeutung :

1. Das Lösungsmedium.

2. Die Applikationstechnik.

3. Das Volumen der applizierten Präparatlösung.

4. Die biologische Konstitution des Bienenvolkes.

Die ersten drei Punkte sind galenische und technische Probleme zur Methodik der Therapie und leicht experimentell erfassbar. Die verhaltensbiologischen Parameter, die die biologische Konstitution eines Bienenvolkes beschreiben, sind hingegen sehr vielschichtig und nur sehr schwer experimentell darzustellen. In dieser Arbeit soll daher nur der saisonale Einfluss auf die Verteilung berücksichtigt werden. Dies war insbesondere nach der Arbeit von RUTTNER et al. (1981) von Interesse, da dort ein Unterschied im Therapieerfolg mit systemischer Chemotherapie der Varroatose bei der Sommerbehandlung und der Winterbehandlung gezeigt werden konnte. Gerade die Unterschiede in der Biologie des Wintervolkes und des Bienenvolkes im Sommer sollten auf die Verteilung eines bestimmten Probenvolumens eine grosse Rolle spielen. 


\section{MATERIAL UND METHODEN}

\section{Sommerversuche}

Als Versuchsvölker wurden brutfreie 6-Wabenableger in Zanderbeuten benutzt. Die Bienen wurden im Flugraum bei $25^{\circ} \mathrm{C}$ und $55 \%$ rel. Feuchte im 12 Stunden-Tag gehalten. Zur quantitativen Darstellung der Präparatverteilung wurde die Methode von Nixon und RibBands (1952) modifiziert angewandt.

Die Testlösungen wurden mit dem $\beta$-Strahler $\mathbf{P}^{32}$ radioaktiv markiert und in Kunststoffolien eingeschweisst oder in einer Einwegspritze an das Volk verabreicht. Nach definierten Zeitabständen wurden von jeder Wabe 50 Bienen und aus 100 Futterzellen pro Volk je $20 \mu 1$ Honig entnommen und die Radioaktivität im Liquid-Scintillation-Counter ausgewertet. Pro Volk wurde eine Dosis von $0,05 \mathrm{mCi}$ appliziert. Das $\mathrm{P}^{32}$ wurde als Orthophosphat in $\mathrm{HCl}$-Lösung $(p \mathrm{H} \mathrm{2-3)}$ angewandt.

Die radioaktive Tracermethodik hat den Nachteil, dass die getesteten Bienenvölker nach Versuchsende in Folge der radioaktiven Kontamination abgetötet werden müssen. Zudem müssen die Versuche in geschlossenen Flugräumen durchgeführt werden, um den Verflug markierter Bienen zu vermeiden.

\section{Winterversuche}

Zur Darstellung der Präparatverteilung in der Wintertraube wurde deshalb eine Farbstoffmarkierungsmethode gewählt. Die Versuchsvölker konnten im Freiland stehen bleiben und brauchten nach Versuchsende nicht vernichtet zu werden. Demgegenüber steht eine etwas aufwendigere Auswertung als bei der $\mathbf{P}^{32}$-Methode, die jedoch nicht zuletzt wegen der fehlenden Umweltbelastung in Kauf genommen wurde.

Die Versuchsvölker besassen in etwa die gleiche Volksstärke wie in den Sommerexperimenten. Als Farbstoff wurde nach Vorversuchen Methylenblau in einer $2 \%$ igen Lösung appliziert. Pro Volk wurde ein Volumen von $50 \mathrm{ml}$ mit der Einwegspritze aufgebracht. In einer Kontrollgruppe wurde nur mit einer reinen Farbstoff-Wasser-Lösung behandelt. In einer zweiten Versuchsgruppe wurden der Methylenblaulösung pro Volk $35 \mathrm{mg}$ Chlordimeformhydrochlorid (K-79) zugegeben, um einen eventuellen Effekt des Akarizides auf die Verteilung aufzuzeigen. Nach $24 \mathrm{~h}$ wurde den Völkern je eine Stichprobe von 150 Bienen entnommen. Es wurde dabei darauf geachtet, möglichst aus allen Bereichen der Wintertraube Bienen zu erhalten. Eine Auftrennung der Proben in verschiedene Regionen der Traube erschien nicht sinnvoll, da der Eingriff in das Bienenvolk zu einer beträchtlichen Unruhe führte und die Bienen sich zum Teil stark durchmischten.

Zur Auswertung wurden die Bienen einzeln in $1,5 \mathrm{ml} 96 \%$ igem Alkohol homogenisiert und anschliessend bei $3000 \mathrm{~g} 10 \mathrm{~min}$ zentrifugiert. Der Überstand, in dem sich das Methylenblau gelöst hat, wird in eine Küvette mit $1 \mathrm{~cm}$ Schichtdicke gegeben und die Extinktion im Photometer bei $600 \mathrm{~nm}$ bestimmt.

Ein Teil der Proben wurde vor der Homogenisation in $96 \%$ igem Alkohol gewaschen, um äusserlich anhaftenden Farbstoff zu entfernen und die Stärke der kutikularen Kontamination zu messen.

\section{ERGEBNISSE}

\section{Sommerversuche}

\subsection{Zeitlicher Verlauf}

Als erstes wurde der zeitliche Verlauf der Präparatverteilung untersucht. Hierzu wurden nach $1,2,4,8,12,24,48,72$ und $120 \mathrm{~h}$ Bienenproben entnommen. Ein Probenvolumen von $200 \mathrm{ml}$ wurde in der geschweissten Kunststoffolie appliziert. Als 
Tracer wurde $\mathrm{P}^{32}$ benutzt. In der Abb. 1 ist der zeitliche Ablauf der Resorption im Bienenvolk dargestellt. Man kann beobachten, dass sich die Verteilung nach $24 \mathrm{~h}$ nicht mehr signifikant verändert.

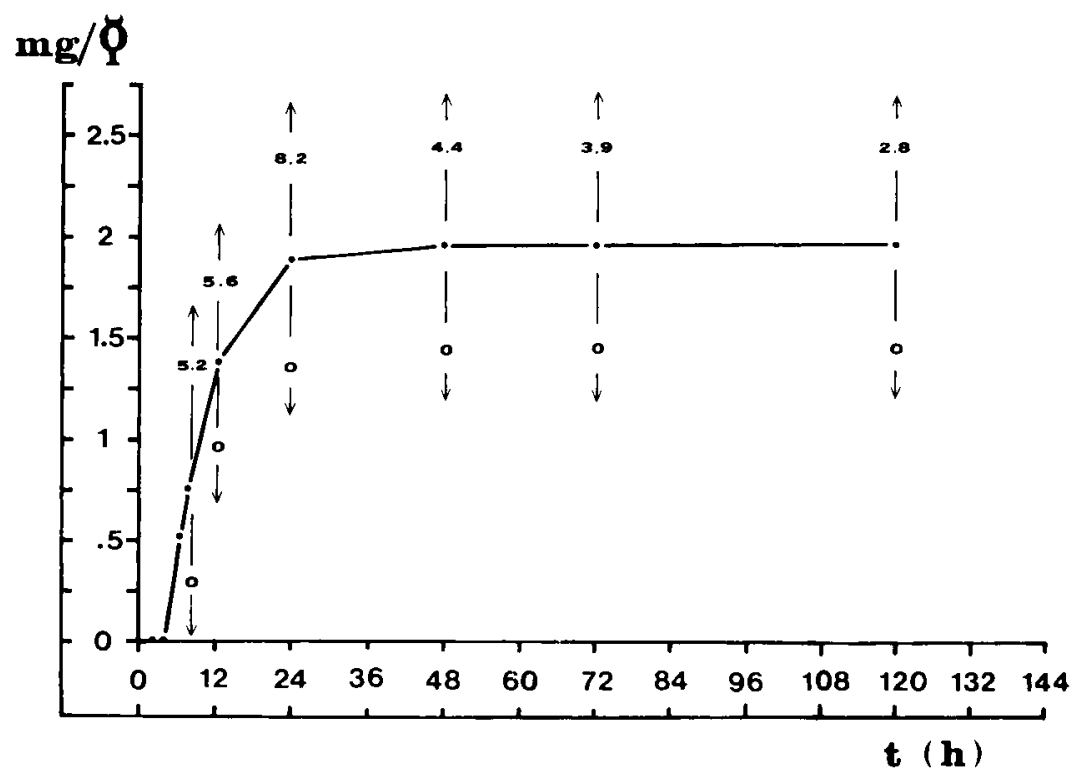

Aвв. 1. - Zeitlicher Verlauf der Präparatverteilung.

Die Kurve gibt die Dichtemittel der Verteilungen bei den entsprechenden Zeiten an. Die Spannweite ist je Messpunkt eingetragen (Probenvolumen $200 \mathrm{ml}$ ).

FIG. 1. - Course of drug distribution.

The graph shows the modes and ranges at the corresponding times

(Test volume $200 \mathrm{ml}$ ).

Dies ist bedeutsam für die weiteren Tests über den Einfluss der Applikationsmethodik auf die Verteilung und zur Darstellung eines Volumeneffektes. Diese Versuche konnten dadurch bereits nach $24 \mathrm{~h}$ beendet werden, ohne ein verfälschtes Bild der Verteilung zu erhalten.

\subsection{Volumeneffekt in Bienen}

Die Verteilung von $50 \mathrm{ml}, 100 \mathrm{ml}, 200 \mathrm{ml}$ und $2 \times 50 \mathrm{ml}$ Proben wurde untersucht. In allen Fällen wurde eine industriell gefertigte Zuckerlösung (Apirève $80 \mathrm{~S}$ ) mit Hilfe der Kunststoffolienmethode appliziert. Abb. 2 zeigt die entsprechenden Verteilungen nach $48 \mathrm{~h}$. Man sieht, dass die Schiefe mit zunehmendem Probenvolumen deutlich abnimmt. Hat die Verteilungskurve bei $50 \mathrm{ml}$ zunächst die Charakteristik einer Poissonverteilung, so wird mit Vergrösserung des Volumens das Histogramm deutlich näher an eine Normalverteilung angeglichen. Bei einer zweimaligen Applikation von je $50 \mathrm{ml} \mathrm{im} \mathrm{Abstand} \mathrm{von} 24 \mathrm{~h}$ wird schliesslich eine Normalverteilung erreicht. Eine 


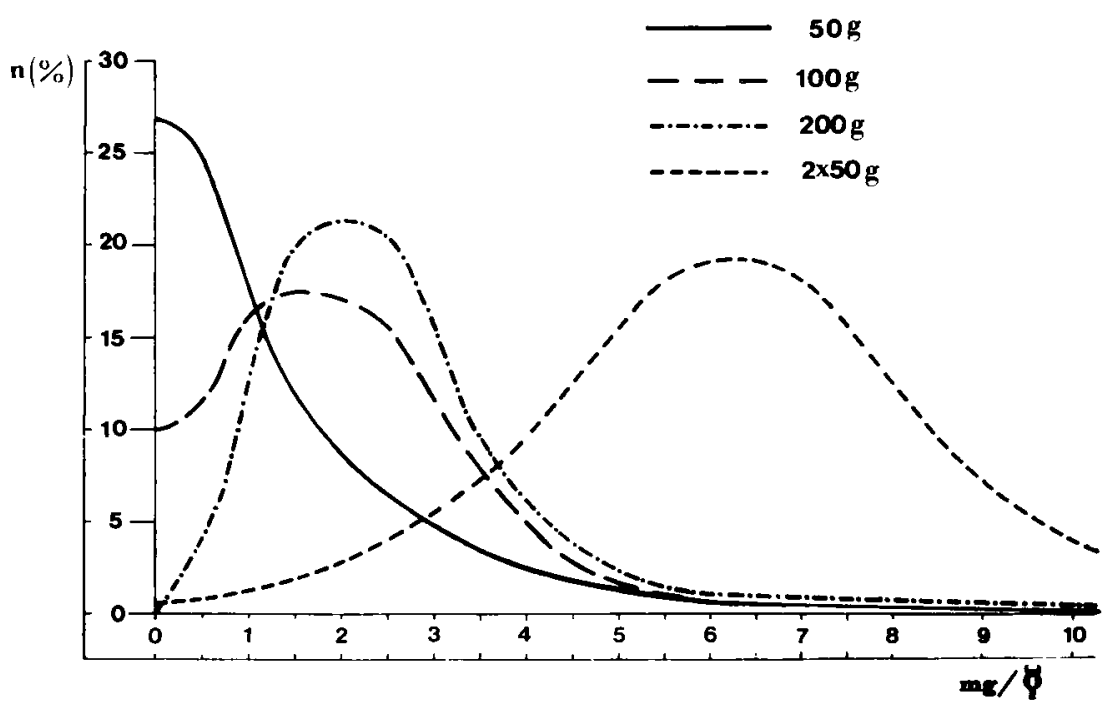

Aвв. 2. - Volumeneffekt von Apirêve $80 \mathrm{~S}$ auf die Präparatverteilung in Bienen. Zur besseren Übersicht wurden die Histogramme idealisiert und als Verteilungskurven dargestellt.

FIG. 2. - The effect of volume of Apirêve $80 \mathrm{~S}$ on drug distribution in honey bees. For clarity the histograms are represented as distributions-graphs.

signifikante Abweichung von der Normalverteilung ist mit dem Test von Kolmogoroff und Smirnoff nicht nachweisbar, wie im Falle der anderen Probenvolumina bei einer einmaligen Applikation.

Es scheint demnach zu einer Zufallsverteilung des Präparats zu kommen, wobei je nach Applikationsweise und Volumen alle Übergänge von der Poissonverteilung bis zur Normalverteilung erreicht werden können.

\subsection{Galenischer Effekt in Bienen}

Es sollte hier gezeigt werden, inwieweit sich eine unterschiedliche Aufbereitung des Präparats auf die Verteilung auswirken kann. Markiertes Wasser und Apirève $80 \mathrm{~S}$ wurden in der Kunststoffolie sowie in der Einwegspritze appliziert. Abb. 3 zeigt die Verteilung in den Bienen nach $48 \mathrm{~h}$ (Tracer $\mathrm{P}^{32}$ ). Bei einem Probenvolumen von $2 \times 50 \mathrm{ml}$ konnte kein signifikanter Unterschied in der Verteilung festgestellt werden, der auf die Applikationsweise zurückgeführt werden kann. Offensichtlich hat hier nur das Volumen einen Einfluss auf die Verteilungskurve.

\subsection{Volumeneffekt im Honig}

Inwieweit Probenmaterial auch in Honigzellen eingelagert wurde, konnte an den Probenvolumina $100 \mathrm{ml}, 200 \mathrm{ml}$ und $2 \mathrm{mal} 50 \mathrm{ml}$ Apirève $80 \mathrm{~S}$, in der Kunststoffolie appliziert, gezeigt werden. In der Abb. 4 sind die entsprechenden Verteilungen graphisch dargestellt. In allen Fällen handelt es sich um angenäherte 


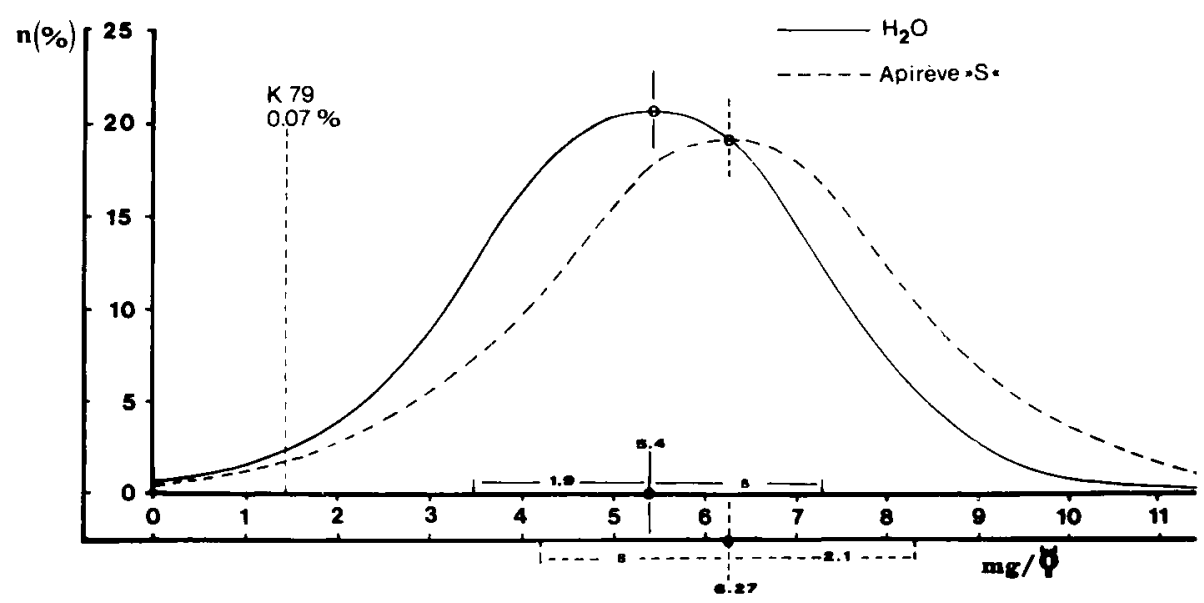

Aвв. 3. - Effekt des Trägermediums auf die Verteilung in Bienen

Die unterbrochene vertikale Linie gibt die $\mathrm{LD}_{50}$ für die Milbe Varroa jacobsoni bei der Applikation einer 0,07\%igen Chlordimeformhydrochlorid-Lösung an $(2 \times 50 \mathrm{ml})$. $n=$ Anzahl Bienen in \% der Gesamtstichprobe.

FIG. 3. - The effect of the medium on drug distribution in honey bees.

The dotted vertical line shows the $\mathrm{LD}_{50}$ for the mite Varroa jacobsoni with a $0.07 \%$ chlordimeforme-hydrochloride-solution treatment $(2 \times 50 \mathrm{ml})$. $n=$ number of bees in $\%$ of the total random sample.

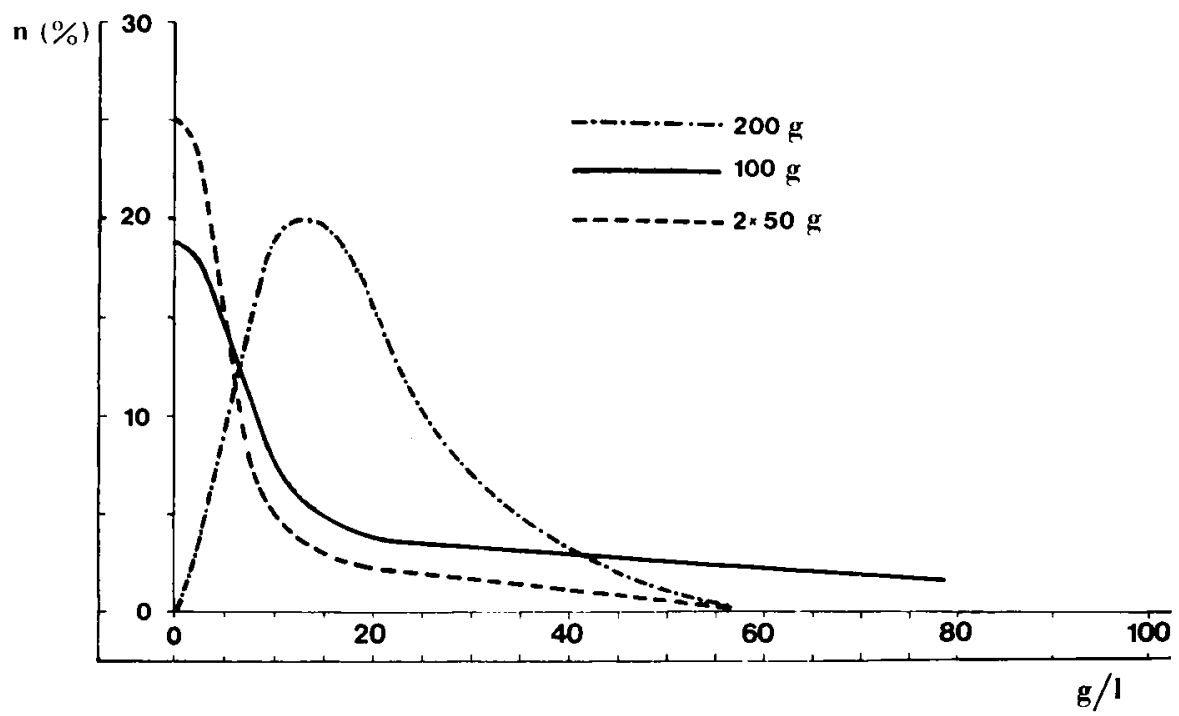

AвB. 4. - Volumeneffekt auf die Präparatverteilung in Honigzellen.

Die Abszisse gibt die Mediumkonzentration im Honig in $\mathrm{g} / \mathrm{l}$ an. $n=$ Anzahl Zellen in \% (Trägermedium : Apirêve $80 \mathrm{~S}$ ).

FIG. 4. - The effect of volume on drug distribution in honey cells. Concentration of medium in honey is given in $\mathrm{g} / \mathrm{l}$. $n=$ number of cells in \% (Medium : Apirêve $80 \mathrm{~S}$ ). 
Poissonverteilungen. Das heisst : Die Einlagerung markierten Materials $\left(\mathrm{P}^{32}\right)$ ist als seltenes Zufallsereignis anzusehen. $\mathrm{Da}$ eine möglichst grosse Häufigkeit für unkontaminierte Zellen bei einer Therapie wünschenswert ist, insbesondere im Hinblick auf eine Rückstandsproblematik, erscheint die Applikationsform mit $2 \times 50 \mathrm{ml}$ am sinnvollsten.

Über ein Viertel der untersuchten Zellen zeigte keine nachweisbare Kontamination. Bei der Gabe von $200 \mathrm{ml}$ waren hingegen alle untersuchten Honigzellen radioaktiv kontaminiert.

\subsection{Galenischer Effekt im Honig}

In dieser Messreihe wird der Effekt des Mediums Wasser im Vergleich zur Zuckerlösung (Apirêve $80 \mathrm{~S}$ ) untersucht. Es wurden $2 \times 50 \mathrm{ml}$ in der Kunststoffolie (Apirêve $80 \mathrm{~S}$ ) bzw. in der Einwegspritze (Wasser) appliziert. In der Abb. 5 kann man sehen, dass die unterschiedliche galenische Aufbereitung auch auf die Verteilung in Honigzellen keinen Einfluss hat. In beiden Fällen sind $25 \%$ der Zellen nicht kontaminiert.

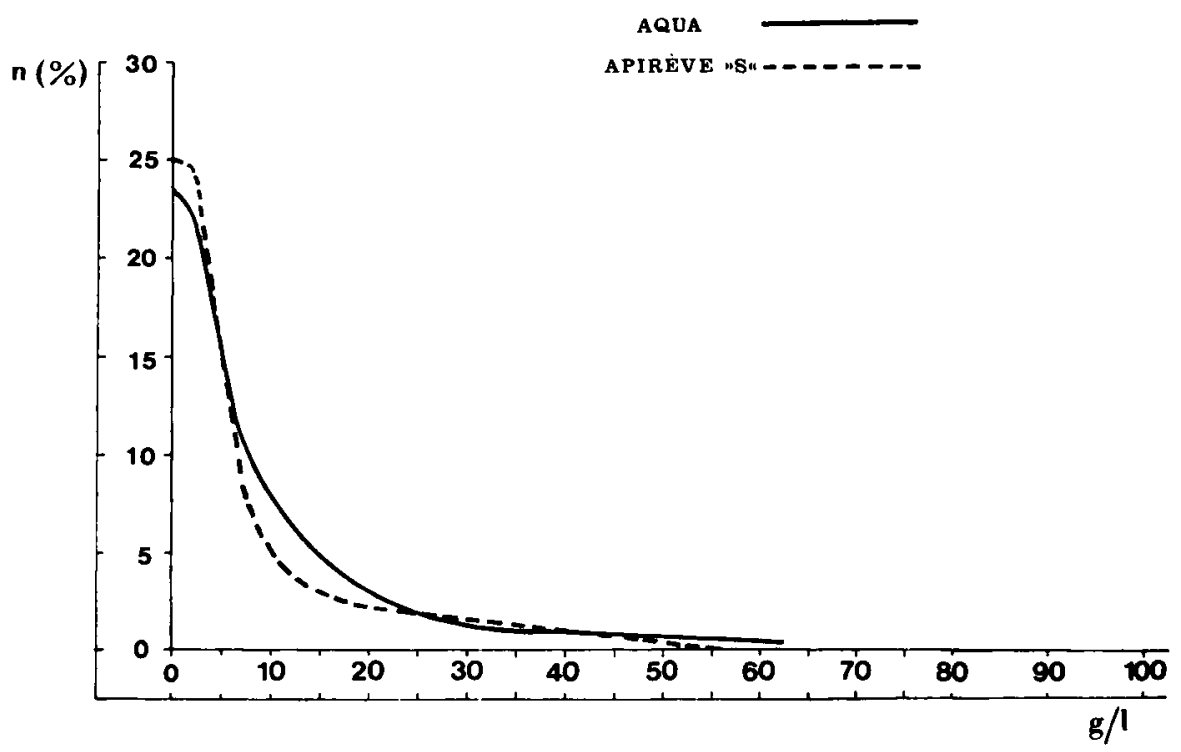

Авв. 5. - Effekt des Trägermediums auf die Verteilung in Honigzellen.

Die Mediumkonzentration ist in $\mathrm{g} / \mathrm{l}$ angegeben. $\mathrm{n}=$ Anzahl Zellen in \%. Testvolumen : $2 \times 50 \mathrm{ml}$.

FIG. 5. - Medium effect on drug distribution in honey cells.

Concentration of medium is given in $\mathrm{g} / \mathrm{l} . \mathrm{n}=$ number of cells in $\%$. Test volume : $2 \times 50 \mathrm{ml}$.

\section{Winterversuche}

Diese Versuche wurden im Freiland mit der Methylenblaumarkierung durchgeführt. 


\section{1. Äusserliche Kontamination an Bienen}

Zur Darstellung der äusserlichen Kontamination mit dem Lösungsmittel wurden $50 \mathrm{ml}$ Methylenblau-markiertes Wasser mit der Einwegspritze auf die Wintertraube geträufelt. Abb. 6 zeigt die verschiedenen Farbstoffmengen bei gewaschenen und

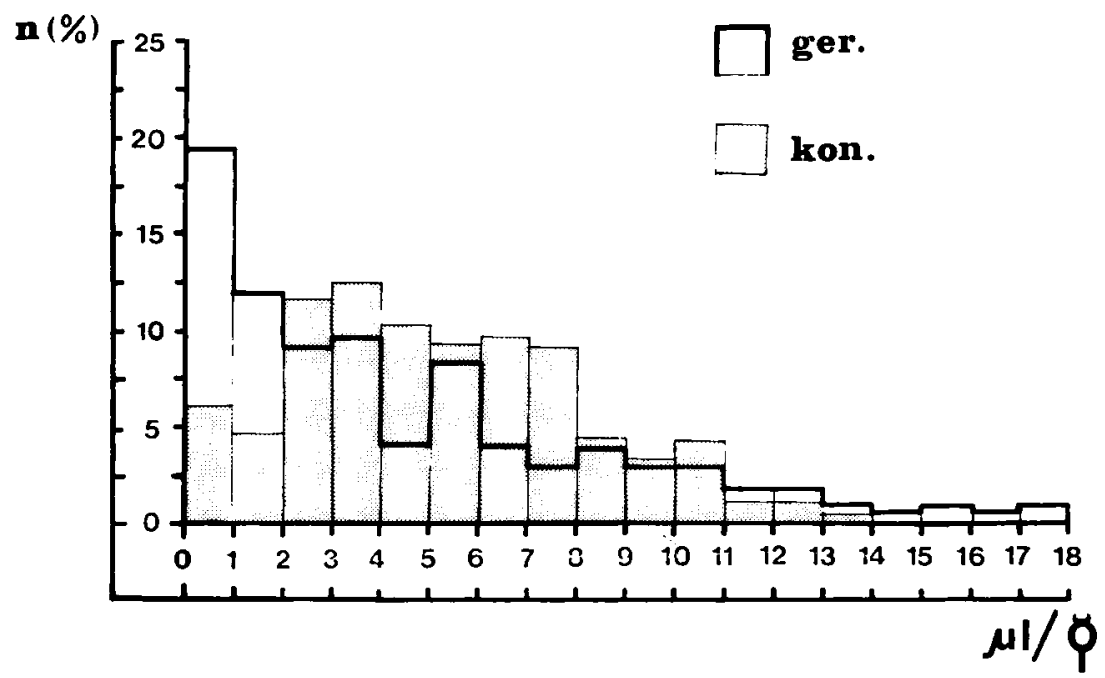

AвB. 6. - Methylenblau-Kontamination an Bienen in der Wintertraube (Testvolumen : $50 \mathrm{ml}$ ). Das aufgenommene Volumen ist in $\mu \mathrm{l} /$ Arbeitsbiene angegeben. $\mathrm{n}=$ Anzahl Bienen in \%; ger. $=$ gereinigt; kon. $=$ kontaminiert.

FIG. 6. - Methylene blue contamination of honey bees in winter clusters (test volume : $50 \mathrm{ml}$ ). Medium intake is given in $\mu 1 /$ honeybee. $\mathrm{n}=$ number of bees in $\%$. ger. $=$ washed. kon $=$ contaminated.

kontaminierten Bienen. Ein Mittelwertvergleich erscheint angesichts der Schiefe der Verteilungen nicht praktikabel. Berechnet man die Dichtemittel, so erhält man eine mittlere äusserliche Kontamination von $2,5 \mu \mathrm{l}$ Probelösung pro Biene. Der parameterfreie Test nach Kolmogoroff und Smirnoff ergibt einen signifikanten Unterschied zwischen gereinigten und nichtgereinigten Bienen auf dem 0,05 Niveau. Mit Hilfe dieser Methode konnte festgestellt werden, dass das systemisch wirksame Präparat K-79, wenn es der Farbstofflösung in therapeutischen Dosen zugesetzt wird, zu einer geringeren äusseren Kontamination führt. Abb. 7 zeigt die Differenz der Häufigkeiten in den einzelnen Klassen zwischen gereinigten und kontaminierten Bienen bei der entsprechenden Behandlung. Man sieht, dass bei der K-79 Applikation die Unterschiede deutlich vermindert sind. Offensichtlich gelingt es den Bienen besser sich von der anhaftenden Lösung zu säubern.

\subsection{Präparateffekt auf die Verteilung in Bienen}

Inwieweit es bei Zugabe von K-79 zur Farbstofflösung tatsächlich zu einer besseren Resorption kommt, zeigt die Abb. 8. Man sieht deutlich, dass ein grösseres 


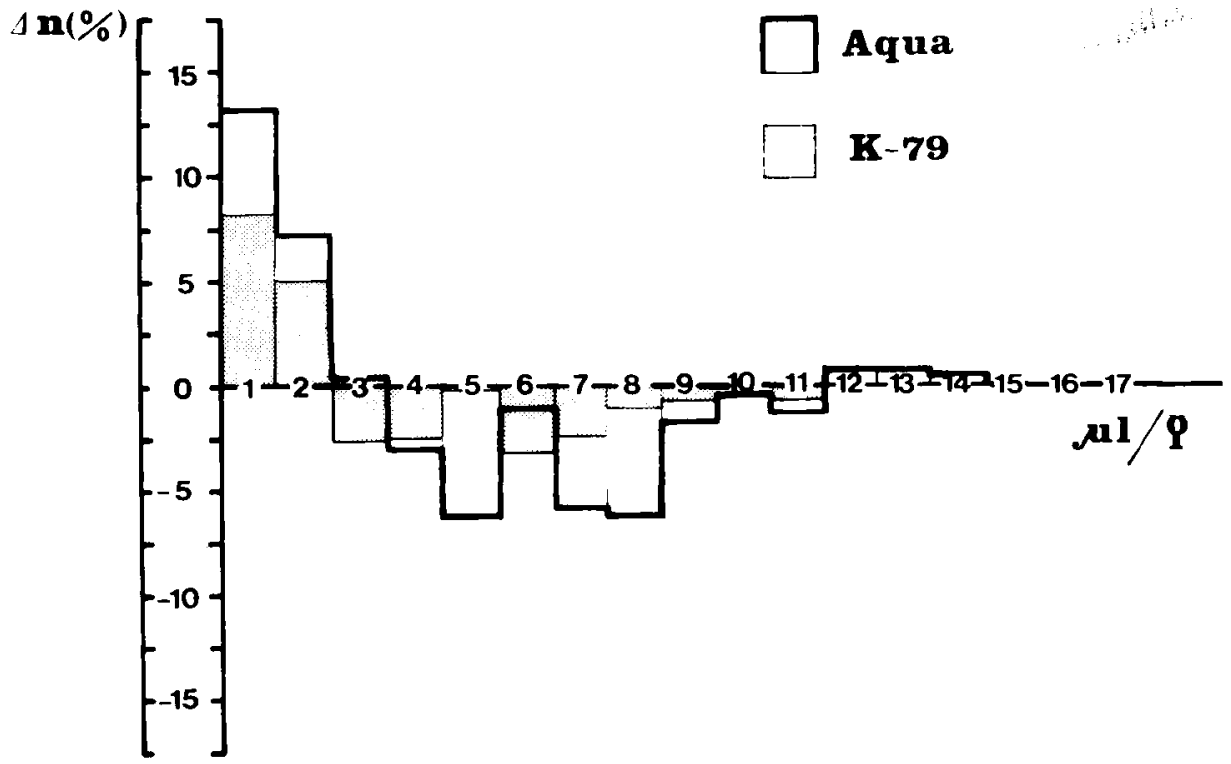

Aвв. 7. - Vergleich der Kontamination: Wasser - K-79

$\mathrm{n}=$ Differenz zwischen der Häufigkeit von gereinigten und kontaminierten Bienen (in \%). Aqua $=$ Wasserbehandlung; K-79 = Chlordimeformhydrochlorid-Behandlung.

FIG. 7. - Comparison of contamination : water - K-79

$\mathrm{n}=$ difference in frequency of washed and contaminated bees in \%. Aqua = water treatment;

$\mathrm{K}-79=$ chlordimeformehydrochloride-treatment.

n

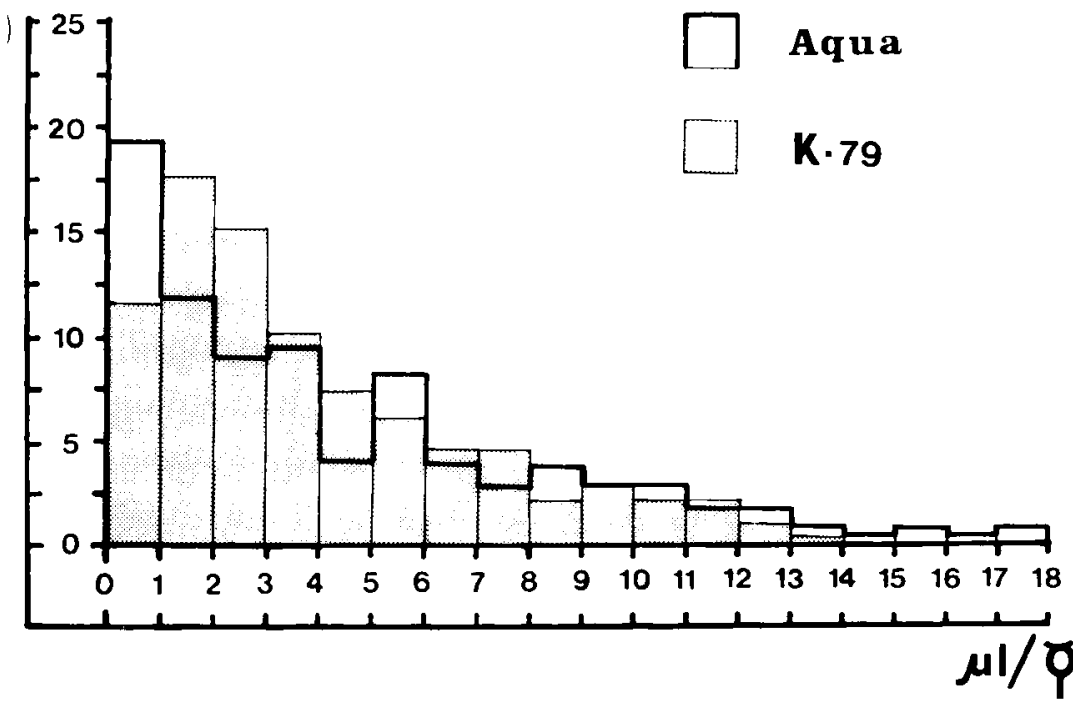

Aв8. 8. - Aufnahme von Präparatlösung und Wasser nach $24 h$ in $\mu$ l pro Arbeitsbiene. $\mathrm{n}=$ Anzahl Bienen in \%; Aqua $=$ Wasserbehandlung $(50 \mathrm{ml})$;

$\mathrm{K}-79=$ Chlordimeformhydrochloridlösung-Behandlung $(50 \mathrm{ml})$.

FIG. 8. - Intake of drug solution and water after $24 \mathrm{~h}$ given in $\mu \mathrm{l} / \mathrm{bee}$. $\mathrm{n}=$ number of bees in $\%$; Aqua = water treatment $(50 \mathrm{ml})$;

$\mathrm{K} \cdot 79=$ chlordimeformehydrochloride treatment $(50 \mathrm{ml})$. 
Volumen von den Bienen aufgenommen wird, wenn man der Farbstofflösung K-79 zusetzt. Die Differenz der Dichtemittel beträgt $0,91 \mu \mathrm{l} /$ Biene. Prüft man die beiden Verteilungen mit dem parameterfreien Verfahren nach Kolmogoroff und Smirnoff, so erhält man einen signifikanten Unterschied auf dem 0,05 Niveau.

\section{Vergleich Sommer-Winter}

Die Unterschiede in der Verteilung von $50 \mathrm{ml}$ Probenvolumen in gleichstarken Völkern unter Winter- und Sommerbedingungen sind in Abb. 9 dargestellt. Das

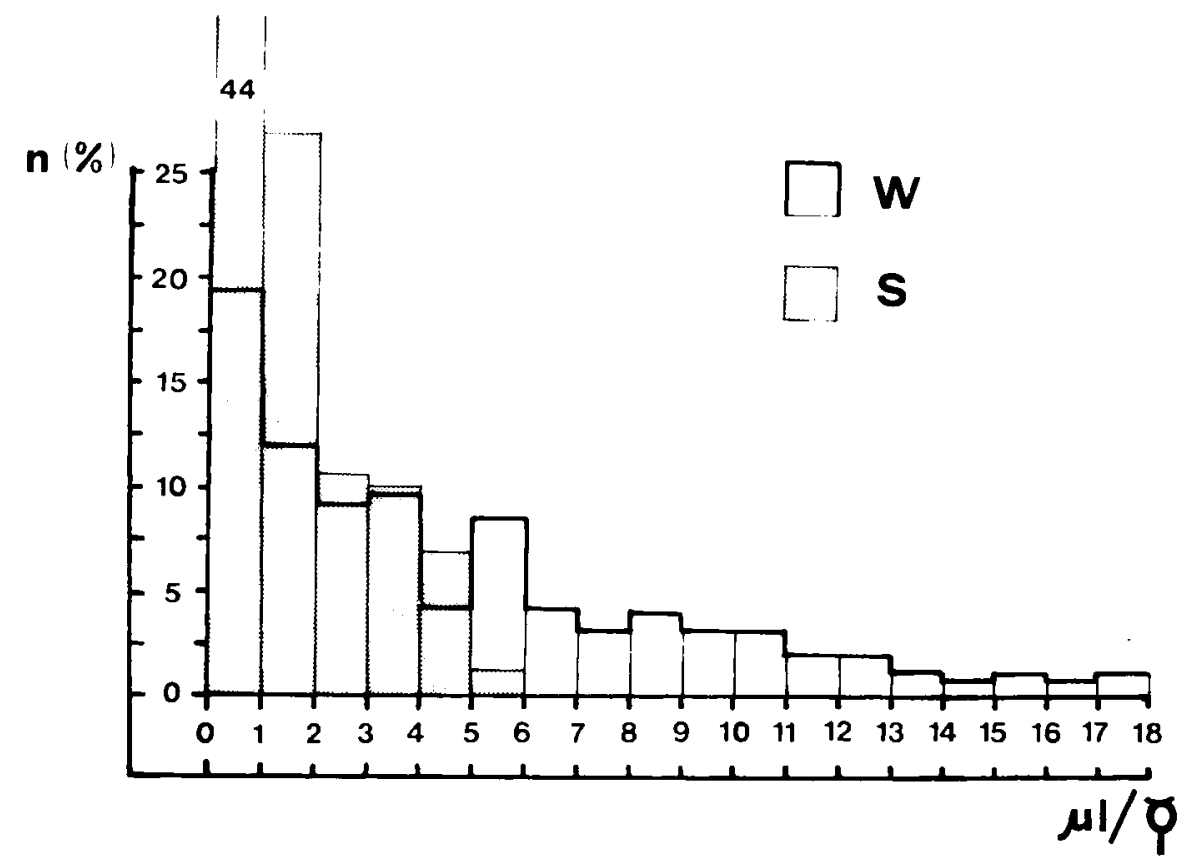

ABB. 9. - Vergleich der Präparatverteilung bei einer einmaligen Applikation von 50 ml Probenvolumen in Sommer- und Wintervölkern. $\mathrm{W}=$ Winter; $\mathrm{S}=$ Sommer; $\mathrm{n}=$ Anzahl Bienen in $\%$.

FIG. 9. - Comparison of drug distribution after a single application of $50 \mathrm{mltest}$ volume in summer and winter colonies. $\mathrm{W}=$ winter colonies; $\mathrm{S}=$ summer colonies; $\mathrm{n}=$ number of bees in $\%$.

Probenvolumen wird in der Wintertraube wesentlich besser aufgenommen und gleichmässiger verteilt als in Sommervölkern. Man kann einen statistisch bedeutsamen Unterschied auf dem 0,001 Niveau nachweisen (Test von Kolmogoroff und Smirnoff). Als Lagemass ist der Interdezilbereich $\mathrm{I}_{\mathbf{8 0}}$, der genau $80 \%$ der Werte beinhaltet, dem Mittelwert oder dem Dichtemittel überlegen. Dieser Bereich beträgt im Sommer $2 \mu 1 /$ Biene, während im Wintervolk ein Interdezilbereich von $14 \mu 1 /$ Biene erreicht wird. Aus Versuchen zur Bestimmung der Toxizität von K-79 auf Varroa jacobsoni ist bekannt, dass bei der angewandten Applikationsweise eine Menge von 1,5 $\mu 1$ aufgenommenen Probenvolumens pro Biene für die Milbe letal ist. In Sommervölkern 
lagen nach einer einmaligen Behandlung mit $50 \mathrm{ml}$ Testvolumen $50 \%$ der Bienen unterhalb dieser kritischen Grenze. In der Wintertraube liegen hingegen nur $25 \%$ unter der milbenletalen Dosis. Man sollte demnach annehmen können, dass nach einer einmaligen Behandlung im Winter $75 \%$ der Milben abgetötet werden.

Extrapoliert man anhand der Mittel und der Varianzen bei den Sommerversuchen von der einmaligen Winterbehandlung auf die Verteilung nach einer zweiten Applikation von $50 \mathrm{ml}$, so erhält man die Kurven der Abb. 10. Man sieht, dass es zu einer Mittelwertverschiebung kommt. Bei geringerer Streuung werden im Sommer im Mittel 5,4 $\mu \mathrm{l} /$ Biene aufgenommen, während im Winter bei grösserer Streuung nahezu das doppelte Volumen mit 10,2 $\mu \mathrm{l} /$ Biene resorbiert wird. Der z-Test zeigt, dass der Mittelwert im Winter auf dem 0,01 Niveau grösser ist als der Wert der Sommerversuche. Betrachtet man die Häufigkeit von Bienen, die auch nach einer zweiten K-79 Applikation eine zu geringe Akarizidmenge aufgenommen haben, so sieht man, dass alleine auf Grund der Präparatverteilung im Sommer 5,5\% der Milben nicht vom Akarizid in therapeutischen Dosen erreicht wird. In Wintervölkern sollte dieser Prozentsatz nur bei $1,98 \%$ liegen, vorausggesetzt, die Milben sind zufällig auf den Bienen verteilt.

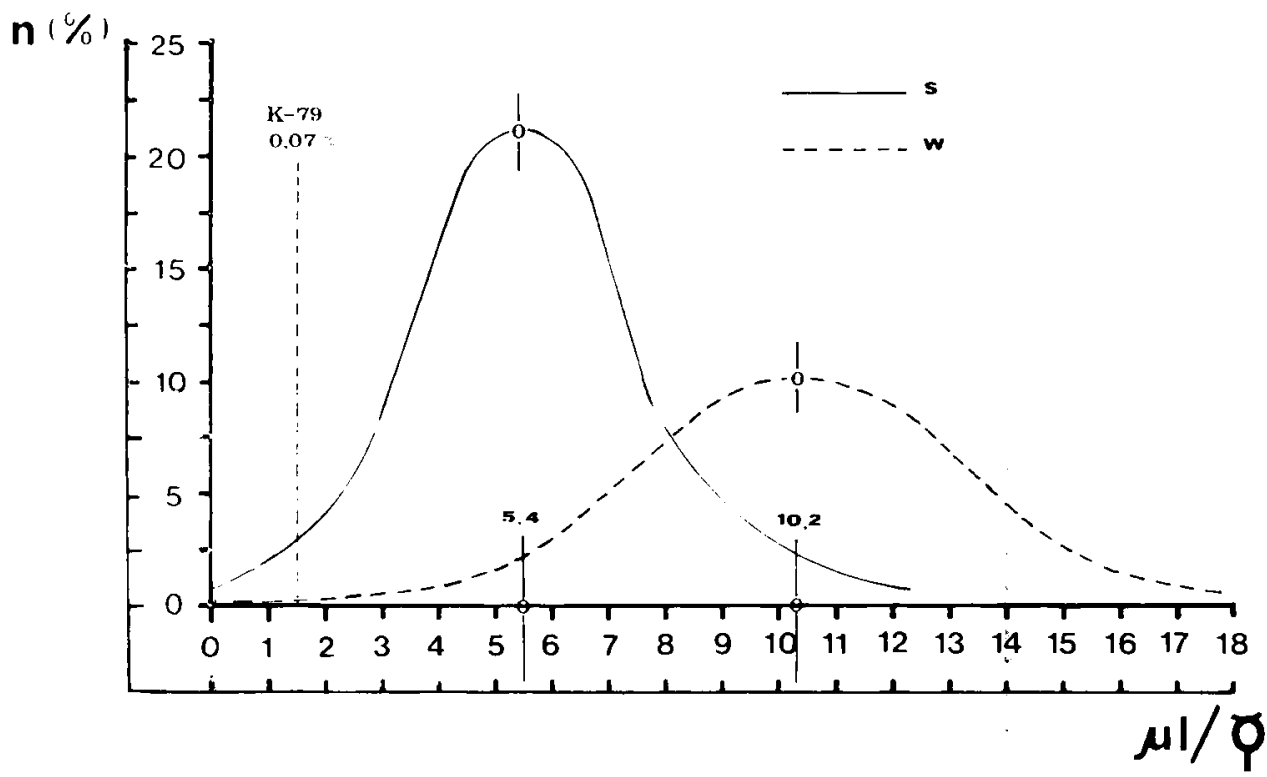

AвB. 10. - Vergleich der Präparatverteilung bei einer zweifachen Applikation von je $50 \mathrm{ml}$ Probenvolumen in Sommer- und Wintervölkern. $\mathrm{W}=$ Winter (hypothetisch); $\mathrm{S}=$ Sommer; $\mathrm{n}=\mathrm{Anzahl}$ Bienen in \%; die unterbrochene vertikale Linie gibt die LD $_{50}$ für die Milbe Varroa jacobsoni bei der Applikation einer 0,07\%igen $\mathrm{K}$-79-Lösung $(2 \times 50 \mathrm{ml})$ an.

FIG. 10. - Comparison of drug distribution after a double application of $50 \mathrm{ml}$ in summer and winter colonies.

$\mathrm{W}=$ Winter (hypothetical); $\mathrm{S}=$ Summer; $\mathrm{n}=$ number of bees in $\%$; the dotted vertical line shows the $\mathrm{LD}_{50}$ for Varroa jacobsoni with a $0.07 \%$ K-79 treatment $(2 \times 50 \mathrm{ml})$. 


\section{DISKUSSION}

Aus den dargelegten Ergebnissen ergeben sich wichtige Hinweise für den Einsatz von systemischen Präparaten im Bienenvolk. Die Experimente zur Darstellung des Volumeneffekts auf die Präparatverteilung im Bienenvolk zeigen, dass eine mehrmalige Behandlung mit kleinen Volumina zu den besten Ergebnissen führt. Im Falle von Poissonverteilungen ist der Anteil der Bienen, die eine zu geringe Präparatmenge erhalten, zu hoch, um einen sinnvollen therapeutischen Erfolg zu garantieren. Nur im Falle der zweimaligen Applikation von $50 \mathrm{ml}$ kann eine Normalverteilung beobachtet werden, bei der im hinreichenden Masse die Bienen vom entsprechenden Präparat erreicht werden. In der Tabelle 1 sind die Daten zusammengefasst, die die zu

TAB. 1. - Mortalität von Varroa jacobsoni bei systemischer Therapie mit K-79 bei unterschiedlicher Applikationsweise.

Die Werte sind anhand der experimentell ermittelten Präparatverteilungen im Bienenvolk errechnet

(LD nach RADEMACHER, 1980).

TABL. 1. - Mortality of Varroa jacobsoni after systemic treatment with $K-79$ using different testvolumes.

Data is calculated acccrding to the experimentally

determined drug distributions.

\begin{tabular}{l|c|c|c|c|c|c}
\hline \hline & \multicolumn{4}{|c|}{$\begin{array}{c}\text { Sommer } \\
\text { Summer }\end{array}$} & \multicolumn{2}{c}{$\begin{array}{c}\text { Winter } \\
\text { Winter }\end{array}$} \\
\hline $\begin{array}{l}\text { Volumen (ml) } \\
\text { Volume } \\
\text { Mortalität in \% } \\
\text { Mortality } \\
\text { Apirêve 80 S }\end{array}$ & 50 & 100 & 200 & $2 \times 50$ & 50 & $2 \times 50$ \\
Wasser & & & & & & \\
Water & 27,1 & 55,2 & 68,4 & 95,1 & - & - \\
\hline \hline
\end{tabular}

erwartende Milbenmortalität im Falle der Chemotherapie der Varroatose mit der entsprechenden Behandlungsmethodik beschreiben. Im Gegensatz zum Volumeneffekt scheint die galenische Aufbereitung des Präparats bei den untersuchten Methoden keinen Einfluss auf die Verteilung zu zeigen. Allerdings konnte Maul (unveröffentlicht) zeigen, dass es bei unterschiedlichen Lösungsmitteln zu einer verschieden schnellen Abnahme des Probenvolumens kommt. Dabei war eine besonders schlechte Abnahme bei der Verabreichung von festen Medien zu beobachten. Bei der Anwendung flüssiger Formulierungen scheint jedoch Medium und Applikationsmethode nur einen untergeordneten Einfluss auf die Verteilung zu haben, vorausgesetzt, das Bienenvolk nimmt die Probe in angemessener Zeit (max. $12 \mathrm{~h}$ ) auf. Ähnliches konnten MoRITZ et al. (1980) mit halbquantitativen Farbmarkierungsmethoden an Sommervölkern nachweisen. Verschiedene Zuckerlösungen mit unterschiedlicher Methodik appliziert, führten zu gleichen Verteilungen, wenn gleiche Volumina verfüttert wurden. 
Die verschiedenen Effekte auf die Einlagerung in Honigzellen stellen sich ähnlich wie die Präparatverteilung in den Bienen dar. Es ist nur ein Volumeneffekt nachweisbar. Erfreulich, dass auch hier die Applikation von $2 \times 50 \mathrm{ml}$ die günstigste Verteilung im Falle von rückstandsproblematischen Präparaten hat. Offensichtlich wird das kleine Volumen kaum in die Zellen eingelagert sondern zu einem Grossteil von den Bienen direkt resorbiert. Dies entspricht einem ähnlichen Effekt wie bei einer Reizfütterung, die der Imker gibt, um sein Bienenvolk zu stimulieren. Das Futter wird resorbiert, aber nicht in die Honigzellen eingelagert.

Die Experimente, die an der Wintertraube durchgeführt wurden, zeigen interessante Abweichungen von den Sommerversuchen. Während im Sommer keine äusserliche Kontamination nachweisbar war, konnte im Winter eine beträchtliche Präparatmenge auf der Bienenkutikula gefunden werden. Offensichtlich ist das Putzverhalten in der Wintertraube stark reduziert. Dennoch ist die Gesamtverteilung durch den engen Zusammenhalt der Wintertraube signifikant besser als in Sommervölkern. Besonders günstig erweist sich bei der Therapie der Varroatose, dass K-79 offensichtlich die Aktivität innerhalb der Traube erhöht, was zu einer noch besseren Resorption des Mittels führt.

Von besonderem Interesse sind die Daten der Abb. 9 und Abb. 10. Vergleicht man diese Zahlen mit den tatsächlich im Freilandversuch (RUTTNER et al. 1981) gefundenen Daten, so findet man erstaunliche Übereinstimmungen, die die hypothetische Verteilung einer zweiten Winterbehandlung recht glaubhaft erscheinen lassen. Aus den Daten der Tabelle 2 sieht man, dass im Feldversuch nach der 2. Behandlung 98,9\%

TAB. 2. - Vergleich der Milbenmortalität im Feldversuch (RUTTNER et al. 1981) und der anhand der Präparatverteilung berechneten hypothetischen Milbenabfallquoten nach einer Behandlung mit $2 \times 50 \mathrm{ml} \mathrm{K-79} \mathrm{Lösung} \mathrm{in} \mathrm{Wasser} \mathrm{(0,07 \% ig).}$

TABL. 2. - Comparison of mite mortality in field tests (RUTTNER et al. 1981) and mite drop number according to drug distribution after a K-79 treatment $(2 \times 50 \mathrm{ml}, 0,07 \%)$.

\begin{tabular}{|c|c|c|c|c|}
\hline & \multicolumn{2}{|c|}{$\begin{array}{l}\text { Milben-Abfallzahlen nach } \\
\text { Mite drop number after }\end{array}$} & \multirow{2}{*}{$\begin{array}{l}\text { Rest } \\
\text { Rest }\end{array}$} & \multirow{2}{*}{$\begin{array}{c}\text { Gesam } \\
\text { Total }\end{array}$} \\
\hline & $\begin{array}{l}\text { 1. Behandlung } \\
\text { 1st treatment }\end{array}$ & $\begin{array}{l}\text { 2. Behandlung } \\
\text { 2nd treatment }\end{array}$ & & \\
\hline $\begin{array}{l}\text { Feldversuch } \\
\text { ( } 73 \text { Völker) }\end{array}$ & 54088 & 18082 & 803 & 72973 \\
\hline $\begin{array}{l}\text { Fieldtest } \\
\text { ( } 73 \text { colonies })\end{array}$ & & & & \\
\hline $\begin{array}{l}\text { Mortalität } \\
\text { Mortality }\end{array}$ & $74,12 \%$ & $24,78 \%$ & $1,1 \%$ & $100 \%$ \\
\hline $\begin{array}{l}\text { Hypothetisch } \\
\text { Hypothetical }\end{array}$ & 54730 & 16798 & 1445 & 72973 \\
\hline $\begin{array}{l}\text { Mortalität } \\
\text { Mortality }\end{array}$ & $75,0 \%$ & $23,02 \%$ & $1,98 \%$ & $100 \%$ \\
\hline
\end{tabular}


der Milben getötet wurden. Die theoretische Modellkurve ermittelt einen Wert von $98,02 \%$ toter Milben.

Zusammenfassend ist festzustellen, dass eine systemische Therapie an Bienenvölkern im Winter grössere Aussichten auf Behandlungserfolg hat als im Sommer. Die Biologie der Honigbiene begünstigt eine solche Therapie im Winter. Berücksichtigt man die Rückstandsproblematik, die wohl bei den meisten solcher Präparate gegeben sein wird, insbesondere dadurch, dass der Honig als Nahrungsmittel eine grosse Rolle spielt, erscheint eine Behandlung von Bienenvölkern mit systemischen Präparaten im Sommer und zur warmen Jahreszeit, in der sich das Bienenvolk nicht zur Traube zusammengezogen hat, nicht empfehlenswert.

\section{RESUMÉ \\ RÉPARTITION DU MEDICAMENT DANS LE TRAITEMENT SYSTÉMIQUE DES ECTOPAR ASITOSES CHEZ APIS MELLIFERA L.}

En chimiothérapie systémique des ectoparasitoses de l'Abeille (Apis mellifica L.) le résultat du traitement dépend largement de la répartition du médicament dans la colonie. Les travaux de KüNZLER (1980) et de RUTTNER et al. (1980) ont fourni les premiers résultats de la lutte contre la varroase par la thérapeutique systémique. Pour suivre la répartition du médicament dans la ruche on a marqué l'excipient avec un traceur radioactif $\left(\mathrm{P}^{32}\right)$ ou avec du bleu de méthylène.

Dans les essais sur les colonies d'été on a étudié l'influence de l'excipient et du volume du produit appliqué sur la répartition (Fig. 1, 2 et 3). On a également décrit la répartition dans les cellules de miel (Fig. 4 et 5). Sur les colonies d'hiver on a montré l'influence de la substance active systémique, l'hydrochlorure de chlordimeform (K-79), sur la répartition (Fig. 6, 7 et 8).

La comparaison entre les colonies d'été et d'hiver montre que la répartition du médicament est nettement plus avantageụse dans la grappe hivernale (Fig. 9 et 10). Tandis qu'en hiver chaque ouvrière prélève en moyenne 10,2 $\mu \mathrm{l}$, en été la moyenne tombe à $5,4 \mu \mathrm{l}$. Si l'on calcule la mortalité des acariens due à la répartition du médicament dans le cas du traitement de la varroase au $\mathrm{K}-79$, on atteint avec un traitement de $50 \mu \mathrm{l}$ de K-79 a $0,07 \%$ effectué deux fois une mortalité de $94,5 \%$ en été, et de $98,02 \%$ en hiver, en admettant une répartition au hasard des acariens sur les abeilles (Tabl. 1). Si l'on compare les valeurs obtenues théoriquement avec les données trouvées par RUTTNER et al. (1981) dans leur expérimentation en champ, on trouve une concordance extrêmement précise. La valeur théorique de la mortalité s'écarte d'environ $0,08 \%$ de la valeur obtenue empiriquement.

D'après ces résultats et en considérant le problème des résidus de médicaments toxiques pour l'Homme, une chimiothérapie systémique pendant la saison chaude, au cours de laquelle la colonie ne s'est pas ramassée en grappe, ne semble pas recommandable.

\section{SUMMARY}

DRUG DISTRIBUTION IN SYSTEMIC THERAPY OF ECTOPARASITOSES OF APIS MELLIFERA L.

In systemic therapy of ectoparasitoses in honey bees (Apis mellifera L.) the treatment result will depend on the drug distribution in the colony. The first experiments of systemic therapy in honey bees were reported by KüNZLER (1980) and RUTTNER et al. (1980) to control varroatosis. A radioactive tracer $\left(\mathrm{P}^{32}\right)$ and methylene-blue was used to trace drug distribution. 
The effect of the carrier medium and applied volume on drug distribution was tested in summer colonies (Fig. 1, 2 and 3). Distribution in honey cells is shown in Fig. 4 and 5. The distribution of the systemic drug K-79 on winter colonies is also shown (Fig. 6, 7 and 8). In comparing summer and winter colonies, drug distribution in the winter cluster is superior (Fig. 9 and 10). Drug intake per worker bee amounts to a mean of $10.2 \mu \mathrm{l}$ in winter out only $5.4 \mu \mathrm{l}$ in summer.

Mite mortality in varroatosis therapy with $\mathrm{K}-79$, considering drug distribution, after a double treatment $(2 \times 50 \mathrm{ml} ; 0,07 \%)$ resulted in a mortality of $94,5 \%$ in summer and of 98.02 in winter (Tabl. 1). Comparing theoretical data with empirical data (RutrNer et al., 1981) the mortality was almost equal to each other, a difference of $0,08 \%$.

Considering these results and the potential problem of drug residues in honey, systemic therapy in the warm season, when bees do not cluster, is not recommendable.

Eingegangen im August 1981. Reçu pour publication en août 1981 .

\section{LITERATURVERZEICHNIS}

GösswaLD K., KLOFT W., 1958. - Radioaktive Isotope zur Erforschung des Staatenlebens der Insekten. Umschau, 24, 743-745.

KüNZLER K., 1980. - Bekämpfung der Varroatose am Bien. Die Biene (5) 1980, 197-198.

MORITz R. F. A., 1981. - Altersabhängige Empfindlichkeit von Varroa jacobsoni Oudemans gegen K-79 (Chlordimeformhydrochlorid). Verhandl. Internat. Symp. "Diagnose und Therapie der Varroatose" 1980 Bad Homburg, Verlag Apimondia Bukarest, 25-32.

Moritz R. F. A., Koeniger N., Maul V., 1981. - Verteilung systemisch wirkender Präparate im Bienenvolk (Apis mellifera L.). Verhandl. Internat. Symp. " Diagnose und Therapie der Varroatose" 1980, Bad Homburg, Verlag Apimondia Bukarest, 62-68.

Nixon H. L., RibBands C. R., 1952. - Food transmission within the honeybee community. Proc. Roy. Soc. B, 140, 43-50.

RADEMACHER E., 1981. - Laborversuche mit K-79 im Einsatz gegen Varroa jacobsoni. Verhandl. Internat. Symp. " Diagnose und Therapie der Varroatose " 1980, Bad Homburg; Verlag Apimondia Bukarest; 155-167.

RutTNER F., RitTer W., Götz W, 1980. - Chemotherapie der Varroatose über die Haemolymphe der Bienen. Allg. Disch. Imker Z., 14 (5), 160-166.

Ruttner F., Wachendörfer G, Valder W. A., Kaiser E., Maul V., Wissen W, Harlander B, Becker W., BotTin F., 1981. - Erfahrungen mit dem Akarizid K-79 (Chlordimeformhydrochlorid) in Hessen zur Bekämpfung der Varroatose der Honigbiene. Dtsch. Tierärztl. Wschr., 88 (5), 161-168. 